\title{
Refractory T Acute Lymphoblastic Leukemia
}

National Cancer Institute

\section{Source}

National Cancer Institute. Refractory T Acute Lymphoblastic Leukemia. NCI Thesaurus.

Code C150510.

T acute lymphoblastic leukemia that does not respond to treatment. 